\title{
CORRECTION
}

\section{Correction to: Repeating a suicide attempt during adolescence: risk and protective factors 12 months after hospitalization}

\author{
Bojan Mirkovic ${ }^{1,2,6}$ (D) David Cohen ${ }^{1,3} \cdot$ Sébastien Garny de La Rivière ${ }^{4} \cdot$ Hugues Pellerin $^{1} \cdot$ Jean-Marc Guilé ${ }^{4,5}$. \\ Angèle Consoli ${ }^{1} \cdot$ Priscille Gerardin $^{6,7}$
}

Published online: 12 March 2020

(c) Springer-Verlag GmbH Germany, part of Springer Nature 2020

\section{Correction to: European Child \& Adolescent Psychiatry https://doi.org/10.1007/s00787-020-01491-x}

In the original article, incorrect Table 1 was published. The correct Table 1 is given below.

Table 1 Sociodemographic and clinical characteristics

\begin{tabular}{|c|c|c|c|c|c|c|}
\hline \multirow[t]{2}{*}{ Variable } & \multicolumn{2}{|c|}{ Baseline $(N=320)$} & \multicolumn{2}{|c|}{6 Months-FU $(N=135)$} & \multicolumn{2}{|c|}{$\begin{array}{l}12 \text { Months-FU } \\
(N=91)\end{array}$} \\
\hline & $n$ & $\%$ & $n$ & $\%$ & $n$ & $\%$ \\
\hline \multicolumn{7}{|l|}{$\operatorname{Sex}$} \\
\hline Boys & 55 & 17.2 & 21 & 15.5 & 12 & 13.3 \\
\hline Girls & 265 & 82.8 & 114 & 84.5 & 78 & 86.7 \\
\hline \multicolumn{7}{|l|}{ Age group (years) } \\
\hline $13-14$ & 141 & 44.0 & 55 & 41 & 43 & 48.1 \\
\hline $15-17$ & 179 & 56.0 & 80 & 59 & 48 & 51.9 \\
\hline \multicolumn{7}{|l|}{ Living arrangement } \\
\hline Without both parents & 187 & 58 & 70 & 52 & 44 & 47.8 \\
\hline With both parents & 133 & 42 & 65 & 48 & 47 & 52.2 \\
\hline Repeated grade at school, Yes & 101 & 31.5 & 48 & 35.8 & 30 & 33.7 \\
\hline Mental health treatment at admission & 100 & 31 & 50 & 37.5 & 31 & 34.8 \\
\hline
\end{tabular}

The original article can be found online at https://doi.org/10.1007/ s00787-020-01491-x.

Bojan Mirkovic

docteur.mirkovic@gmail.com

1 Department of Child and Adolescent Psychiatry, University Hospital Pitié-Salpêtrière AP-HP, University Pierre and Marie Curie, 47-83 Boulevard de l'Hôpital, 75013 Paris, France

2 Unité de Recherche EA4047 (HANDIReSP), University of Versailles Saint-Quentin-en-Yvelines, Versailles, France

3 CNRS, UMR 7222, Institut des Systèmes Intelligents et Robotiques, University Pierre and Marie Curie, Paris, France
$4 \quad$ Child and Adolescent Psychiatry Services, University Hospital of Amiens, University Picardie Jules Verne, Amiens, France

5 Department of Psychiatry, McGill University, Montreal, Canada

6 Department of Child and Adolescent Psychiatry, University Hospital Charles-Nicolle/CH-Rouvray, University of Normandie, Rouen, France

7 Laboratoire CRFDP, University of Normandie, Rouen, France 


\begin{tabular}{|c|c|c|c|c|c|c|}
\hline \multirow[t]{2}{*}{ Variable } & \multicolumn{2}{|c|}{ Baseline $(N=320)$} & \multicolumn{2}{|c|}{6 Months-FU $(N=135)$} & \multicolumn{2}{|c|}{$\begin{array}{l}12 \text { Months-FU } \\
(N=91)\end{array}$} \\
\hline & $n$ & $\%$ & $n$ & $\%$ & $\bar{n}$ & $\%$ \\
\hline \multicolumn{7}{|l|}{ Axis I diagnoses (DSM-IV-R) } \\
\hline Major depressive disorders & 131 & 41.0 & 60 & 44.4 & 37 & 40 \\
\hline Adjustment disorder with depressed mood & 106 & 33.5 & 51 & 38 & 32 & 35 \\
\hline Anxiety disorders & 88 & 28.0 & 34 & 25.4 & 22 & 24.7 \\
\hline Psychotic disorders & 3 & 1 & 0 & 0 & 0 & 0 \\
\hline Disruptive and oppositioal behaviour & 66 & 21.0 & 35 & 26 & 21 & 23 \\
\hline Attention deficit hyperactivity disorder & 17 & 5.0 & 6 & 4.7 & 6 & 6.5 \\
\hline \multicolumn{7}{|l|}{ Borderline disorder (Ad-DIB) } \\
\hline Yes & 224 & 70 & 102 & 75 & 66 & 74.2 \\
\hline No & 89 & 28 & 33 & 25 & 23 & 25.8 \\
\hline \multicolumn{7}{|l|}{ Method of suicide attempt } \\
\hline Intoxication & 242 & 75.6 & 107 & 79 & 69 & 74.9 \\
\hline Laceration & 20 & 6.2 & 10 & 7.5 & 8 & 8.8 \\
\hline Strangulation & 28 & 8.7 & 9 & 6.7 & 4 & 4.4 \\
\hline Precipitation & 13 & 4.0 & 4 & 2.8 & 2 & 2.2 \\
\hline Others & 17 & 5.5 & 5 & 4 & 8 & 8.8 \\
\hline \multicolumn{7}{|l|}{ Number of suicide attempts } \\
\hline 1 & 208 & 65 & 84 & 62 & 54 & 59 \\
\hline 2 & 59 & 18.4 & 26 & 19 & 21 & 23 \\
\hline 3 & 26 & 8.1 & 8 & 6 & 6 & 7 \\
\hline \multirow[t]{2}{*}{$>3$} & 27 & 8.5 & 17 & 13 & 10 & 11 \\
\hline & Mean & $\mathrm{SD}$ & Mean & $\mathrm{SD}$ & Mean & $\mathrm{SD}$ \\
\hline Age at first suicide attempt & 14.63 & 1.42 & 14.7 & 1.28 & 14.3 & 1.32 \\
\hline
\end{tabular}

Ad-DIB = Abbreviated Self-Questionnaire of the Diagnostic Interview for Borderline Personality Disorder 